\title{
Production of the heaviest charged Higgs boson in 3-3-1 models
}

\section{Fredy Ochoa*, R. Martinez and C. Alvarado}

Universidad Nacional De Colombia, Crr. 45 No. 26-85, Bogotá, Colombia

E-mail: faochoap@unal.edu.co, remartinezm@unal.edu.co

\begin{abstract}
We study the production cross section of the heaviest hypercharge-two Higgs boson $\left(H_{2}^{ \pm}\right)$predicted by the 3-3-1 model. Taking into account intermediate vector bosons, including a new $Z^{\prime}$ neutral boson, we calculate the cross section of $H_{2}^{ \pm}$pair production at CERN-LHC hadron collider. Considering $Z^{\prime}$-mass of the order of $1 \mathrm{TeV}$, we found that the cross sections decreases from $100 \mathrm{fb}$ to $1 \times 10^{-3} \mathrm{fb}$ for the $H_{2}^{ \pm}$-mass range $200-1000 \mathrm{GeV}$. We also found that for masses below the kinematic threshold $(500 \mathrm{GeV})$, the cross section splits into three branches for $H_{2}^{ \pm}$-boson, the hipercharge-one $H_{1}^{ \pm}$-boson, and the $H_{2 H D M}^{ \pm}$-boson from a two Higgs doublet model.
\end{abstract}

36th International Conference on High Energy Physics,

July 4-11, 2012

Melbourne, Australia

${ }^{*}$ Speaker. 


\section{Introduction}

An interesting alternative to extend the SM are the models with gauge symmetry $S U(3)_{c} \otimes$ $S U(3)_{L} \otimes U(1)_{X}$, also called 3-3-1 models, which introduce a family nonuniversal $U(1)$ symmetry $[1,2,3]$. These models have a number of phenomenological advantages. First of all, from the cancellation of chiral anomalies and asymptotic freedom in QCD, the 3-3-1 models can explain why there are three fermion families. Secondly, since the third family is treated under a different representation, the large mass difference between the heaviest quark family and the two lighter ones may be understood. Finally, these models contain a natural Peccei-Quinn symmetry, necessary to solve the strong-CP problem. In particular, these models extend the scalar sector of the SM into three $S U(3)_{L}$ scalar triplets. After the spontaneous breaking of the gauge symmetry and rotations into mass eigenstates, the model contains 4 massive charged Higgs $\left(H_{1}^{ \pm}, H_{2}^{ \pm}\right)$, one neutral CP-odd Higgs $\left(A^{0}\right), 3$ neutral CP-even Higgs $\left(h^{0}, H_{1}^{0}, H_{2}^{0}\right)$, and one complex neutral Higgs $\left(H_{3}^{0}\right)$ bosons. In particular, the charged sector is composed of two types of Higgs bosons: hypercharge-one Higgs bosons $H_{1}^{ \pm}$which exhibit tree-level couplings with the SM particles [4], and hypercharge-two Higgs $H_{2}^{ \pm}$ bosons which show couplings with the SM matter through mixing with non-SM particles. In this work we study the $H_{1,2}^{ \pm}$-boson production at CERN-LHC in $p p \rightarrow H_{2}^{+} H_{2}^{-}$pair production. For comparison purposes, we include the $H_{2 H D M}^{ \pm}$-boson production from the two Higgs doublet model.

\section{The 331 spectrum}

We consider a 3-3-1 model where the electric charge is defined by:

$$
Q=T_{3}-\frac{1}{\sqrt{3}} T_{8}+X
$$

with $T_{3}=\frac{1}{2} \operatorname{Diag}(1,-1,0)$ and $T_{8}=\left(\frac{1}{2 \sqrt{3}}\right) \operatorname{Diag}(1,1,-2)$. In order to avoid chiral anomalies, the model introduces in the fermionic sector the following $\left(S U(3)_{c}, S U(3)_{L}, U(1)_{X}\right)$ left- and righthanded representations:

$$
\begin{aligned}
& Q_{L}^{1}=\left(\begin{array}{c}
U^{1} \\
D^{1} \\
T^{1}
\end{array}\right)_{L}:(3,3,1 / 3),\left\{\begin{array}{c}
U_{R}^{1}:\left(3^{*}, 1,2 / 3\right) \\
D_{R}^{1}:\left(3^{*}, 1,-1 / 3\right) \\
T_{R}^{1}:\left(3^{*}, 1,2 / 3\right)
\end{array}\right. \\
& Q_{L}^{2,3}=\left(\begin{array}{c}
D^{2,3} \\
U^{2,3} \\
J^{2,3}
\end{array}\right)_{L}:\left(3,3^{*}, 0\right),\left\{\begin{array}{c}
D_{R}^{2,3}:\left(3^{*}, 1,-1 / 3\right) \\
U_{R}^{2,3}:\left(3^{*}, 1,2 / 3\right) \\
J_{R}^{2,3}:\left(3^{*}, 1,-1 / 3\right)
\end{array}\right. \\
& L_{L}^{1,2,3}=\left(\begin{array}{c}
v^{1,2,3} \\
e^{1,2,3} \\
\left(v^{1,2,3}\right)^{c}
\end{array}\right)_{L}:(1,3,-1 / 3),\left\{\begin{array}{c}
e_{R}^{1,2,3}:(1,1,-1) \\
N_{R}^{1,2,3}:(1,1,0)
\end{array}\right.
\end{aligned}
$$

where $U_{L}^{i}$ and $D_{L}^{i}$ for $i=1,2,3$ are three up- and down-type quark components in the flavor basis, while $v_{L}^{i}$ and $e_{L}^{i}$ are the neutral and charged lepton families. The right-handed sector transforms 
as singlets under $S U(3)_{L}$ with $U(1)_{X}$ quantum numbers equal to the electric charges. In addition, we see that the model introduces heavy fermions with the following properties: a single flavor quark $T^{1}$ with electric charge 2/3, two flavor quarks $J^{2,3}$ with charge $-1 / 3$, three neutral Majorana leptons $\left(v^{1,2,3}\right)_{L}^{c}$ and three right-handed Majorana leptons $N_{R}^{1,2,3}$. On the other hand, the scalar sector introduces one triplet field with $\operatorname{VEV}\langle\chi\rangle_{0}=v_{\chi}$, which provides the masses to the new heavy fermions, and two triplets with VEVs $\langle\rho\rangle_{0}=v_{\rho}$ and $\langle\eta\rangle_{0}=v_{\eta}$, which give masses to the SM fermions at the electroweak scale. The $\left(S U(3)_{L}, U(1)_{X}\right)$ group structure of the scalar fields are:

$$
\begin{aligned}
& \chi=\left(\begin{array}{c}
\chi_{1}^{0} \\
\chi_{2}^{-} \\
\frac{1}{\sqrt{2}}\left(v_{\chi}+\xi_{\chi} \pm i \zeta_{\chi}\right)
\end{array}\right):(3,-1 / 3) \\
& \rho=\left(\begin{array}{c}
\rho_{1}^{+} \\
\frac{1}{\sqrt{2}}\left(v_{\rho}+\xi_{\rho} \pm i \zeta_{\rho}\right) \\
\rho_{3}^{+}
\end{array}\right):(3,2 / 3) \\
& \eta=\left(\begin{array}{c}
\frac{1}{\sqrt{2}}\left(v_{\eta}+\xi_{\eta} \mp i \zeta_{\eta}\right) \\
\eta_{2}^{-} \\
\eta_{3}^{0}
\end{array}\right):(3,-1 / 3) .
\end{aligned}
$$

In particular, the $\rho$ and $\eta$ triplets contain hipercharge-one and hypercharge-two fields. After the symmetry breaking, the above weak eigenstates rotate into the following mass eigenstates $[5,6]$ :

$$
\begin{aligned}
& \text { Hipercharge-one Higgs : } H_{1}^{ \pm}=-S_{\beta_{T}} \rho_{1}^{ \pm}+C_{\beta_{T}} \eta_{2}^{ \pm} \\
& \text {Hipercharge-two Higgs : } H_{2}^{ \pm} \approx \rho_{3}^{ \pm},
\end{aligned}
$$

where $T_{\beta_{T}}=v_{\eta} / v_{\rho}$. The photon $A$, neutral weak boson $Z$ and a new neutral boson $Z^{\prime}$ are [7]:

$$
\begin{aligned}
& A_{\mu}=S_{W} W_{\mu}^{3}+C_{W}\left(\frac{1}{\sqrt{3}} T_{W} W_{\mu}^{8}+\sqrt{1-\frac{1}{3}\left(T_{W}\right)^{2}} B_{\mu}\right), \\
& Z_{\mu}=C_{W} W_{\mu}^{3}-S_{W}\left(\frac{1}{\sqrt{3}} T_{W} W_{\mu}^{8}+\sqrt{1-\frac{1}{3}\left(T_{W}\right)^{2}} B_{\mu}\right), \\
& Z_{\mu}^{\prime}=-\sqrt{1-\frac{1}{3}\left(T_{W}\right)^{2}} W_{\mu}^{8}+\frac{1}{\sqrt{3}} T_{W} B_{\mu},
\end{aligned}
$$

where the Weinberg angle is defined as $S_{W}=\sqrt{3} g_{X} / \sqrt{3 g_{L}^{2}+4 g_{X}^{2}}$, with $g_{L}$ and $g_{X}$ the coupling constants of the groups $S U(3)_{L}$ and $U(1)_{X}$, respectively.

\section{The couplings}

For the interaction between the SM-quarks $q$ and neutral gauge bosons, we found [7]:

$$
\mathscr{L}^{N C}=e Q_{q} \bar{q} \not A q+\frac{g_{L}}{2 C_{W}} \bar{q}\left[\gamma_{\mu}\left(g_{v}^{q}-g_{a}^{q} \gamma_{5}\right) Z^{\mu}+\gamma_{\mu}\left(\widetilde{g}_{v}^{q}-\widetilde{g}_{a}^{q} \gamma_{5}\right) Z^{\prime \mu}\right] q
$$




\begin{tabular}{|c|c|c|c|c|}
\hline Fermion & $g_{v}^{q}$ & $g_{a}^{q}$ & $\widetilde{g}_{v}^{q}$ & $\widetilde{g}_{a}^{q}$ \\
\hline \hline$D^{1}$ & $-\frac{1}{2}+\frac{2}{3} S_{W}^{2}$ & $-\frac{1}{2}$ & $\frac{-1}{6} \sqrt{3-4 S_{W}^{2}}$ & $\frac{-1}{2 \sqrt{3-4 S_{W}^{2}}}$ \\
\hline$D^{m}$ & $-\frac{1}{2}+\frac{2}{3} S_{W}^{2}$ & $-\frac{1}{2}$ & $\frac{3-2 S_{W}^{2}}{6 \sqrt{3-4 S_{W}^{2}}}$ & $\frac{1-2 S_{W}^{2}}{2 \sqrt{3-4 S_{W}^{2}}}$ \\
\hline$U^{1}$ & $\frac{1}{2}-\frac{4}{3} S_{W}^{2}$ & $\frac{1}{2}$ & $\frac{-3-2 S_{W}^{2}}{6 \sqrt{3-4 S_{W}^{2}}}$ & $\frac{-1+2 S_{W}^{2}}{2 \sqrt{3-4 S_{W}^{2}}}$ \\
\hline$U^{m}$ & $\frac{1}{2}-\frac{4}{3} S_{W}^{2}$ & $\frac{1}{2}$ & $\frac{3-8 S_{W}^{2}}{6 \sqrt{3-4 S_{W}^{2}}}$ & $\frac{1}{2 \sqrt{3-4 S_{W}^{2}}}$ \\
\hline
\end{tabular}

Table 1: Vector and Axial couplings of SM quarks and Neutral Gauge Bosons. The index $m=2,3$ labels the $3^{*}$ multiplets.

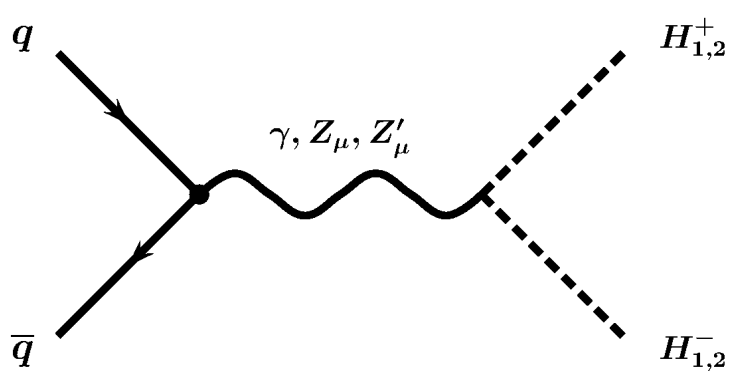

Figure 1: Charged Higgs boson pair production in $p p$ collisions

where $q$ is $U=\left(U^{1}, U^{2}, U^{3}\right)$ or $D=\left(D^{1}, D^{2}, D^{3}\right)$ for up- and down-type quarks, respectively, and $Q_{q}$ the electric charge in units of the positron charge $e$. The vector and axial-vector couplings of the $Z$ and $Z^{\prime}$ bosons are written in table 1 for each SM quark.

On the other hand, from the kinetic term of the Higgs Lagrangian, we obtain the following Higgs-Higgs-Vector interaction associated with the charged Higgs sector::

$$
\begin{aligned}
i \mathscr{L}^{H H V} & =-i e\left[H_{1}^{+} H_{1}^{-}+H_{2}^{+} H_{2}^{-}\right](p-q)^{\mu} A_{\mu} \\
& -\frac{i g_{L}}{2 C_{W}}\left[C_{2 W} H_{1}^{+} H_{1}^{-}+2 S_{W}^{2} H_{2}^{+} H_{2}^{-}\right](p-q)^{\mu} Z_{\mu} \\
& +\frac{i g_{X}}{2 \sqrt{3} T_{W}}\left[\left(C_{2 \beta_{T}}+T_{W}^{2}\right) H_{1}^{+} H_{1}^{-}+2\left(1+T_{W}^{2}\right) H_{2}^{+} H_{2}^{-}\right](p-q)^{\mu} Z_{\mu}^{\prime}
\end{aligned}
$$

\section{Results}

For the pair production from figure 1, we use the couplings in (3.2). Figure 2 show the cross section for Higgs boson pair production $H_{2}^{ \pm}$for $M_{Z}^{\prime}=1 \mathrm{TeV}$ and $p p$ collisions at CM energy of 14 $\mathrm{GeV}$. For comparison purposes, we include the charged Higgs boson $H_{1}^{ \pm}$and $H_{2 H D M}^{ \pm}$from a two 


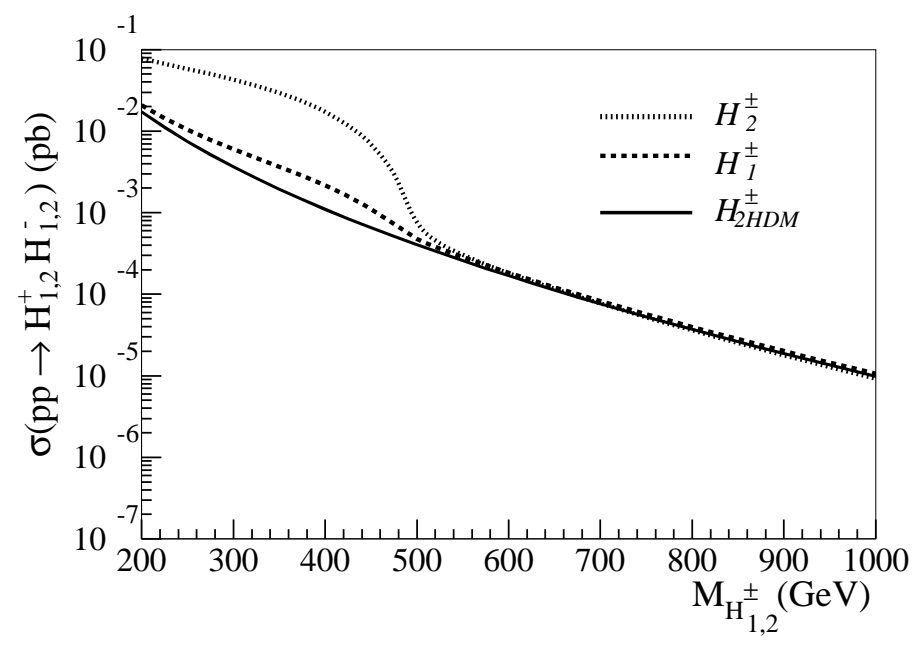

Figure 2: Pair production cross section of charged Higgs bosons

Higgs doublet model (2HDM). We observe the following behaviour:

- Below the kinematic threshold $M_{H}<500 \mathrm{GeV}$, the cross sections split into three branches, where $\sigma\left(H_{2}\right)>\sigma\left(H_{1}\right)>\sigma\left(H_{2 H D M}\right)$.

- The $H_{2}^{ \pm}$bosons exhibit larger contributions than $H_{1}^{ \pm}$. This splits is due to the different contributions exhibits by the $H_{1}^{ \pm}$and $H_{2}^{ \pm}$bosons with the gauge bosons in Eqn. (3.2).

- For 2HDM model, we obtain smaller production ratios due to the fact that in this model the $Z^{\prime}$ contribution does not exist.

- For $M_{H}>500 \mathrm{GeV}$, the $Z^{\prime}$ contribution is suppressed by kinematic conditions $\left(M_{Z}^{\prime}<2 M_{H}\right)$. Thus, the cross section decreases, as shown.

- The $H_{1}^{ \pm}$- $Z^{\prime}$ coupling is $\beta_{T}$-dependent as shown in Eq. (5). For numerical purposes we fix $T_{\beta_{T}}=9$.

\section{Acknowledgements}

This work was supported by Colciencias

\section{References}

[1] F. Pisano and V. Pleitez, $S U(3) \otimes U(1)$ model for electroweak interactions, Phys. Rev. D 46 (1992) 410.

[2] P.H. Frampton, Chiral dilepton model and the flavor question, Phys. Rev. Lett. 69 (1992) 2889. 
[3] R. Foot, H.N. Long and T.A. Tran, $S U(3)_{L} \otimes U(1)_{N}$ and $S U(4)_{L} \otimes U(1)_{N}$ gauge models with right-handed neutrinos Phys. Rev. D 50 (1994) R34.

[4] A. Alves, E. Ramirez Barreto, and A.G. Dias, Production of charged Higgs bosons in a 3-3-1 model at the CERN LHC Phys. Rev. D 84 (2011) 075013.

[5] Rodolfo A. Diaz, R. Martinez, and F. Ochoa, Scalar sector of the $S U(3)_{c} \otimes S U(3)_{L} \otimes U(1)_{X}$ model, Phys. Rev. D 72 (2005) 035018.

[6] Fredy Ochoa and R. Martinez, Family dependence in $S U(3)_{c} \otimes S U(3)_{L} \otimes U(1)_{X}$ models, Phys. Rev. D 72 (2005) 035010.

[7] A. Carcamo, R. Martinez, and F. Ochoa, Z and Z' decays with and without FCNC in 331 models, Phys. Rev. D 73 (2006) 035007. 